\title{
Performing movement sequences with knowledge of results under different visual conditions in adults with Down syndrome
}

\author{
Naznin Virji-Babul',2, Jennifer E.V. Lloyd' and Geraldine Van Gyn ${ }^{3}$ \\ 'Down Syndrome Research Foundation, Vancouver, B.C., Canada \\ ${ }^{2}$ Department of Psychology, University of Victoria, Victoria B.C,. Canada \\ ${ }^{3}$ School of Physical Education, University of Victoria, Victoria B.C., Canada
}

\begin{abstract}
The purpose of this study was to examine the learning of movement sequences in individuals with Down syndrome under different visual information conditions. 10 adults with Down syndrome and 14 neurologically typical adults performed a sequence of movements under two different visual information conditions: full visual feedback of the limb and environment and no visual feedback of the limb. Participants were given knowledge of results of their total movement time after each trial. The entire task was presented as a game and movement time information was given as a "score" after each trial. Participants were also given verbal encouragement throughout the task. As expected, individuals with Down syndrome had significantly slower reaction and movement times than neurologically typical participants. Interestingly, however, mean reaction and movement time was not affected by the visual condition, in either group. Participants with Down syndrome improved their performance over the presented trials, in both visual information conditions. These findings indicate that providing knowledge of results of movement performance can facilitate the performance and coordination of movement sequences even under conditions where visual information of the moving limb is restricted.
\end{abstract}

Keywords: Down syndrome, visual feedback, knowledge of results, movement sequences

\section{Introduction}

One of the features associated with Down syndrome is poor motor coordination or clumsiness (Frith \& Frith, 1974). Although clumsiness is a broad term that is not well defined (Latash, 2000), there are certain motor features that give an overall impression of poor coordination or clumsiness. These include a variable and delayed pattern of motor development, deficits in tasks involving balance, speed and visuo-motor control (Connolly \& Michael, 1986; Harris, 1983), bilateral coordination (Jobling \& Mon-Williams, 2000), and hypotonia (Anson, 1992). Detailed analyses of movements have shown that individuals with Down syndrome demonstrate longer reaction times in comparison with neurologically typical individuals (Blais \& Kerr, 1986; Henderson, Illingworth \& Allen, 1991), longer overall movement times (Hodges, Cunningham, Lyons, Kerr \& Elliott, 1995), and increased variability in performance (Welsh \& Elliot, 2000).
A number of hypotheses have been posed to explain the motor behaviour of individuals with Down syndrome. Frith and Frith (1974) attributed motor clumsiness to a general deficit in developing motor programs. Others have attributed these changes to more specific impairments of somatosensory function (Chiarenza \& Stagi, 2000), impaired decision making processes (Almeida, Corcos \& Hasan, 2000) or impairments in the timing of motor sequences (Henderson et al., 1991; Chiarenza \& Stagi, 2000). To date, the study of motor behaviour in individuals with Down syndrome has been focused largely on evaluation of pointing movements to a target, tapping movements, or single button or key presses. Few studies have examined how individuals with Down syndrome learn and perform continuous movements that require a specific sequence of movements. Elliot, Weeks and Gray (1990) found that verbal-motor problems are more pronounced when oral and manual gestures involve two and three movement components. Jobling and Mon-Williams (2000) found poor per- 
formance on tasks that required asymmetrical activities or tasks that required complex sequences of movement. These findings raise a number of interesting issues. First, is poor performance related to difficulties in coordinating and combining separate movement components, or as Almeida, Marconi, et al. (2000) have suggested, are individuals with Down syndrome simply unable to maintain a certain level of concentration required to complete the task?

A second issue involves the role of visual information in coordinating movements in individuals with Down syndrome. Few studies have directly examined how vision of the moving limb influences the organisation of movement. It is well known that in order to reach to a visual target, information about the extent and direction of the movement must be transformed into a plan expressing changes in joint angles and torques for displacing the hand through its intended hand trajectory (Flanders \& Herrmann, 1992). This transformation from extrinsic to intrinsic coordinates necessitates learning the dynamic properties of the limb (Goodbody \& Wolpert, 1998; Shadmehr \& Mussa-Ivaldi, 1994). While it is generally accepted that contextual information about the state and configuration of the limb is critical for developing an internal representation of limb dynamics (Ghez, Gordon \& Ghilardi, 1995; Ghez \& Sainburg, 1995), the role of visual and proprioceptive (i.e. awareness of limb position) information in learning and maintaining an internal model of limb dynamics is unclear.

Hodges et al. (1995) used a computerised aiming task to evaluate the role of visual feedback in individuals with Down syndrome. Participants were required to move a cursor to a target under two conditions: with and without visual feedback of the cursor. Removal of cursor feedback led to significantly longer movement times and larger errors suggesting that individuals with Down syndrome are highly dependant on visual feedback. Analysis of the movement trajectories appeared to indicate that individuals with Down syndrome used proprioceptive feedback when vision was not available, although unsuccessfully.

The present study was undertaken to examine the learning of a movement sequence by individuals with Down syndrome under different visual information conditions. In particular, we were interested in studying the impact of the visual context after allowing all individuals to have adequate practice to ensure that the task requirements were well understood, and to provide verbal feedback of the movement outcome.

\section{Method}

\section{Participants}

Participating in this study were 10 adults with Down syndrome and 14 neurologically typical adults (Table 1). Neurologically typical adults were recruited from the University of Victoria. Adults with Down syndrome were recruited via the local Down syndrome support group in Victoria, BC. Of the 10 participants with Down syndrome

\begin{tabular}{|l|l|l|}
\hline & Down syndrome & Neurologically typical \\
\hline Age & $20-40$ years & $20-40$ years \\
\hline Mean Age & 30.7 years & 27.57 years \\
\hline Gender & 4 males, 6 females & 5 males, 9 females \\
\hline
\end{tabular}

Table I. Summary of Participants

9 lived either in a group home or with a parent/caregiver. One individual lived independently. Of the 10 participants with Down syndrome 6 were involved in part-time employment and all were involved in some kind of physical activity (i.e. swimming, bowling, walking, etc) at least once a week. All participants provided informed consent before participation in this study according to the guidelines provided by the University of Victoria's Ethics Committee.

\section{Apparatus and task}

Participants were seated facing a button board. Sixteen buttons were arranged into four columns and four rows on a square board. Each button on the board was linked to a $\mathrm{PC}$ and a computer routine was used to provide reaction time (RT) and movement time (MT) of each button press. Yellow luminescent paint was applied to each of the buttons to ensure that participants could see the buttons when the room was darkened. A diagram representing the button board and the sequence of buttons to be depressed was placed on the wall above the button board. Luminescent paint was applied to the diagram so that the participants could see the diagram when the room was darkened. The effects of visual information on reaction time and movement time were examined in two conditions. In the full visual feedback (FVF) condition, the button board and the participants' arms and hands were fully visible. In the no visual feedback (NVF) condition, the room was darkened and only the buttons on the board were visible. Participants could not see their arms and hands.

\section{Procedure}

At the start of each session, instructions were given about the task and the task was demonstrated. Each participant began with practice trials. Five buttons were included in the practice sequence (Figure 1). Each participant began by depressing the start button with his or her dominant hand. The computer then emitted two audible tones. The first tone was provided to alert the participant the trial

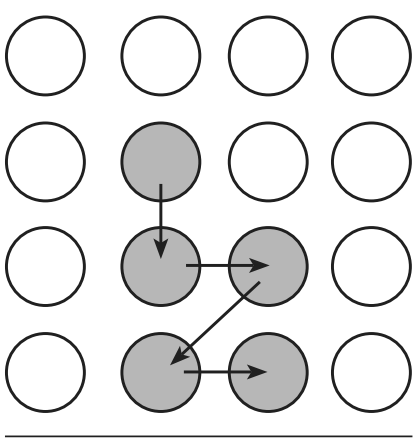

Figure I: Diagram of Practice Sequence was about to begin. Participants were instructed to start the sequence of button pushes immediately upon hearing the second tone. Once the second tone was heard, the timer began, and each participant removed his or her hand from the start button and depressed and released buttons as quickly and accurately as possible. 


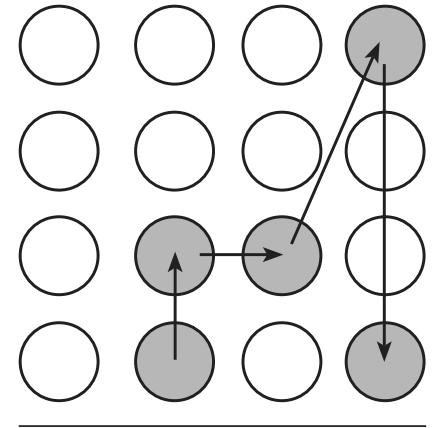

Figure 2: Diagram of Test Sequence
Each participant performed 20 practice trials, 10 with full vision and 10 with no visual feedback. Participants were given knowledge of results of their total movement time (in seconds) after each trial. The entire task was presented as a game and movement time information was given as a "score" after each trial. Participants were also given verbal encouragement throughout the task. For example: "Well done! You did it in 2 seconds!" Following a rest period, two blocks of experimental trials were completed with a new sequence with five buttons (Figure 2) under each visual feedback condition ( 20 trials/block). The order of conditions randomly varied from individual to individual. All participants had the same button sequence. The duration of time between the first and second tones varied between each of the forty trials to ensure participants could not anticipate the start of the timer. Participants were given rest periods between each block of trials.

\section{Data and statistical analysis}

Reaction time was measured from the onset of the second tone to the start of first button release. Movement time was measured from the release of the first button to the depression of the last button. Data from correct trials only were analysed. A 2 (group) x 2 (feedback condition) mixed ANOVA with repeated measures was used to analyse the results.

\section{Results}

\section{Mean reaction time was not affected by the visual condition}

Figure 3, with data in Table 2, shows the mean reaction time under each visual feedback condition for participants with Down syndrome and neurologically typical participants. As expected, individuals with Down syndrome had significantly slower reaction times than neurologically typical participants, $\mathrm{F}_{(1,22)}=340 \mathrm{p}<.001$. Mean reaction times for individuals with Down syndrome were approximately $200 \mathrm{msec}$ longer than neurologically typical participants. There was however, no significant effect of feedback condition $\mathrm{F}_{(1,22)}=.004$, nor an interaction between group and feedback, $\mathrm{F}_{(1,22)}=.192$.

\section{Mean movement time was not affected by the visual condition}

The mean movement times for all participants are shown in Figure 4 and Table 3. On average, neurologically typical participants completed the sequence task in approximately l second. Individuals with Down syndrome took approximately 3 seconds to complete the task. There was a signifi-

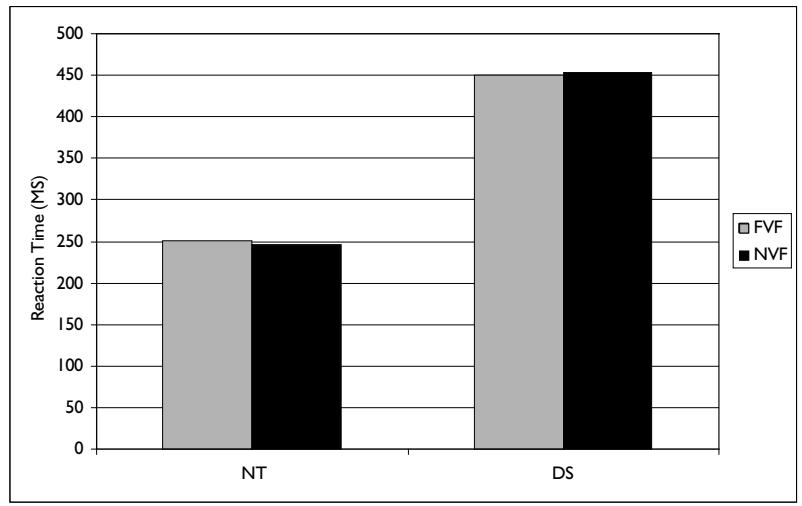

Figure 3: Mean reaction time as a function of group and visual feedback condition.

\begin{tabular}{|l|lr|lr|}
\hline & \multicolumn{2}{|c|}{ Full Visual Feedback } & \multicolumn{2}{|c|}{ No Visual Feedback } \\
\hline & Mean & SD & Mean & SD \\
\hline DS & 450 & 197 & 453 & 193 \\
\hline NT & 251 & 71 & 246 & 70 \\
\hline
\end{tabular}

Table 2: Reaction time (ms) by group and feedback condition

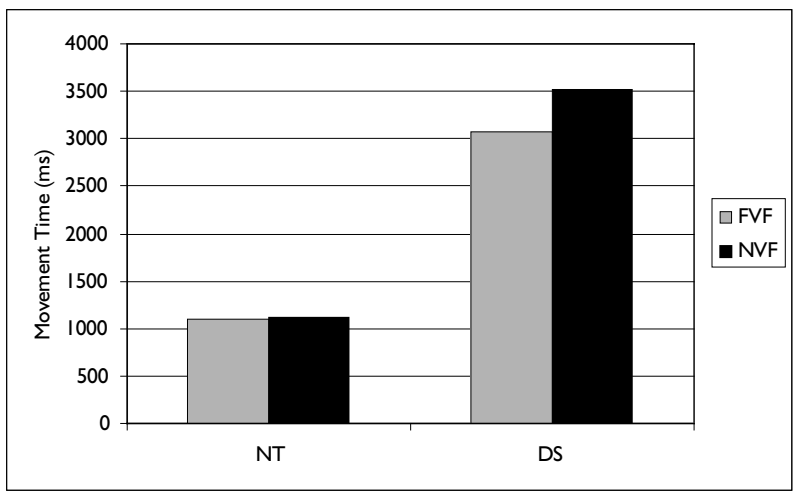

Figure 4: Mean movement time as a function of group and visual feedback condition.

\begin{tabular}{|l|lc|lr|}
\hline & \multicolumn{2}{|c|}{ Full Visual Feedback } & \multicolumn{2}{c|}{ No Visual Feedback } \\
\hline & Mean & SD & Mean & SD \\
\hline DS & 3077 & 993 & 3513 & 1628 \\
\hline NT & 1096 & 244 & 1110 & 260 \\
\hline
\end{tabular}

Table 3. Movement time (ms) by group and feedback condition

cant effect of group, $\mathrm{F}_{(1,22)}=193 \mathrm{p}<.001$. Although overall movement time was less in the FVF condition, there was no significant effect of the feedback condition $\mathrm{F}_{(1,22)}=3.06$, nor an interaction between group and feedback, $\mathrm{F}_{(1,22)}=2.72$. 


\section{Movements of individuals with Down syndrome showed improvements in performance over time in both visual conditions}

In order to examine whether individuals with Down syndrome were able to use the feedback provided to improve their performance, inspection of the mean movement time over the 20 trials was conducted. Figure 5 shows the mean movement time for all individuals with Down syndrome in each visual feedback condition. Note that overall, movement time decreased as a function of practice in all conditions. Participants with Down syndrome were clearly able to focus on the task and use the information about their individual movement time to decrease their movement time. No change in movement time was observed across the 20 trials in neurologically typical participants.

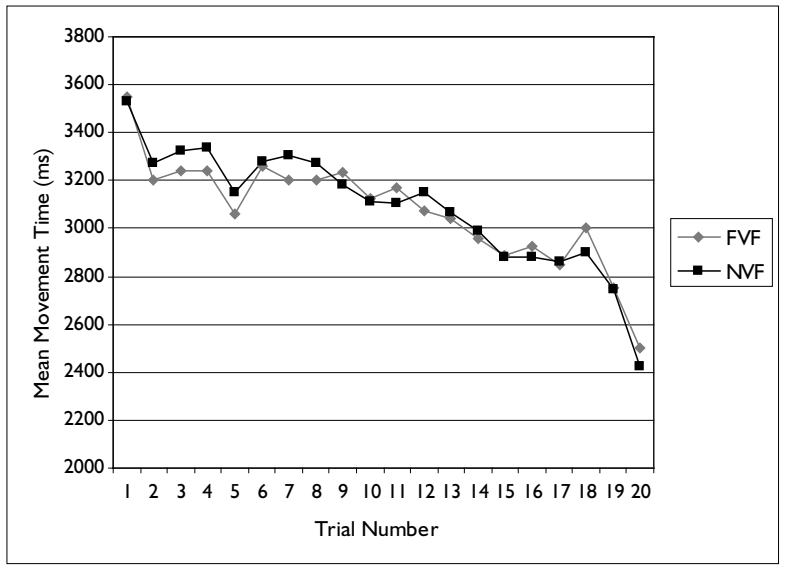

Figure 5: Mean movement time as a function of trial and visual feedback condition for all individuals with Down syndrome.

\section{Discussion}

In this study we first examined the performance of a sequence of movements in adults with Down syndrome. Our results indicated that, with appropriate feedback, individuals with Down syndrome were able to improve their movement times under all conditions. This finding is interesting in that it contradicts some reports that individuals with Down syndrome are often unable to complete tasks even with verbal feedback/encouragement. Frith and Frith (1974) for example, reported that children with Down syndrome often stopped before the end of the task and that verbal prompting led to individuals applying more pressure during a finger tapping task rather than improving movement speed. This highlights the importance of providing appropriate, goal-orientated feedback. In our study verbal feedback and encouragement was based on giving knowledge of results of the overall movement time and verbal encouragement to improve this score in a "game" like environment. This obviously helped to maintain the motivation of individuals. If the goal is not well understood valid assumptions cannot be made about the resulting observed behaviour. Latash and Corcos (1991) have shown also that adults with Down syndrome show improvements in tasks involving single joint movements as they became more confident in their abilities. We have now shown that such improvements can also occur in tasks involving multi-joint movement in a more complex sequence of movements.

It should be noted that the adults with Down syndrome were still showing a downward trend of decreasing movement time by the end of the last trial. Future studies should allow practice until no further decrease in movement time is demonstrated.

A second finding of this study is that adults with Down syndrome were not dependant on visual feedback of the moving limb for coordinating movement sequences. This suggests that in the absence of visual information about the limb, proprioceptive feedback provides sufficient information to coordinate goal directed movements. Almeida, Corcos, et al. (2000) have recently shown that in contrast to patients with known proprioceptive deficits, the movement paths of individuals with Down syndrome are characterised by sharp reversals in direction, similar to that of neurologically typical individuals. This study argues against a proprioceptive deficit in individuals with Down syndrome. The question of whether individuals with Down syndrome are able to develop appropriate internal models, particularly for coordinating multi joint movements, requires much more detailed investigations. Future studies will focus on understanding multi-joint, sequences of movements within a functional context and will directly examine the types of verbal feedback that are most useful to individuals with Down syndrome.

\section{Acknowledgements}

The authors would like to acknowledge all the participants in the study as well as Dr. Kimberly Kerns for help with statistical analysis.

\section{Correspondence}

Naznin Virji-Babul • Centre for Human Movement Analysis, Queen Alexandra Centre for Children's Health, 2400

Arbutus Road, Victoria, B.C., Canada V8N IV7 - Email: naznin@dsrf.org・Tel: +I-250-72I-6849

\section{References}

Almeida, G.L., Corcos, D.M. \& Hasan, Z. (2000). Horizontal-plane arm movements with direction reversals performed by normal individuals and individuals with Down syndrome. Journal of Neurophysiology, 84, 1949-1960.

Almeida, G.L., Marconi, N.F., Tortoza, C., Ferreira, S.M.S., Gottlieb, G.L. \& Corcos, D.M. (2000). Sensorimotor deficits in Down syndrome: Implications for facilitating motor performance. In: D.J. Weeks, R. Chua \& D. Elliott (Eds.), Perceptual-motor behavior in Down syndrome (pp. 151-174). Champaign, IL: Human Kinetics.

Anson, J.G. (1992). Neuromotor control and Down syndrome. In J.J. Summers (Ed.), Approaches to the study of motor control and learning (pp.387-412). Amsterdam, Elsevier Science. 
N. Virji-Babul, J.E.V. Lloyd \& G. Van Gyn • Performing movement sequences under different visual conditions

Bastian, A.J., Martin, T.A., Keating, J.G. \& Thach, W.T. (1996). Cerebellar ataxia: Abnormal control of interaction torques across multiple joints. Journal of Neurophysiology, 76, 492-509.

Blais, C. \& Kerr, R. (1986). Probability information in a complex task with respect to Down syndrome. Journal of Human Movement Studies, 12, 183-194.

Brandt, B.R. \& Rosen, I. (1995). Impaired peripheral somatosensory function in children with Down syndrome. Neuropediatrics, 26, 310-312.

Chiarenza, G.A. \& Stagi, P. (2000). Neurophysiological correlates of perceptual-motor behavior in Down syndrome. In: D.J. Weeks, R. Chua \& D. Elliott (Eds.), Perceptual-motor behavior in Down syndrome (pp. 321-347). Champaign, IL: Human Kinetics.

Connolly, B.H. \& Michael, B.T. (1986). Performance of retarded children with and without Down syndrome, on the Bruininks-Oseretsky test of motor proficiency. Physical Therapy, 66, 344-348.

Elliot, C., Weeks, D.J. \& Gray, S. (1990). Manual and oral praxis in adults with Down's syndrome. Neuropsychologia, 28, 1307-1315.

Flanders, M. \& Herrmann, U. (1992). Two components of muscle activation: Scaling with the speed of arm movement. Journal of Neurophysiology. 67, 931-943.

Frith, U. \& Frith, C.D. (1974). Specific motor abilities in Down's syndrome. Journal of Child Psychology and Psychiatry, 15, 293-301.

Ghez, C. \& Sainburg, R. (1995). Proprioceptive control of interjoint coordination. Canadian Journal of Physiology and Pharmacology, 73, 273-284.

Ghez, C., Gordon, J. \& Ghilardi, M.F. (1995). Impairments of reaching movements in patients without proprioception. II. Effects of visual information on accuracy. Journal of Neurophysiology, 73, 361-372.

Goodbody, S.J. \& Wolpert, D.M. (1998). Temporal and amplitude generalization in motor learning. Journal of Neurophysiology, 79, 1825-1838.

Jobling, A. \& Mon-Williams, M. (2000). Motor Development in Down syndrome: A longitudinal perspective. In: D.J. Weeks, R. Chua \& D. Elliott (Eds.), Perceptual-motor behavior in Down syndrome (pp. 225-248). Champaign, IL: Human Kinetics.

Harris, S.R. (1983). Comparative performance levels of female and male infants with Down syndrome. Physical and Occupational therapy in Pediatrics, 3, 15-21.

Henderson, S.E., Illingworth, S.M. \& Allen, J. (1991). Prolongation of simple manual and vocal reaction times in Down syndrome. Adapted Physical Activity Quarterly, $8,234-241$.

Hodges, N.J., Cunningham, S.J., Lyons, J., Kerr, T.L. \& Elliot, D. (1995). Visual feedback processing and goaldirected movement in adults with Down syndrome. Adapted Physical Activity Quarterly, 12, 176-186.

Latash, M.L. (2000). Motor coordination in Down syndrome: The role of adaptive changes. In: D.J. Weeks, R. Chua \& D. Elliott (Eds.), Perceptual-motor behavior in Down syndrome (pp. 199-223). Champaign, IL: Human Kinetics.

Latash, M.L. \& Corcos, D.M. (1991). Kinematic and electromyographyic characteristics of single-joint movements of individuals with Down syndrome. American Journal of Mental Retardation, 96, 189-201.

Sainburg, R.L., Ghilardi, M.F., Poizner, H. \& Ghez, C. (1995). Control of limb dynamics in normal subjects and patients without proprioception. Journal of Neurophysiology, 73, 820-835.

Shadmehr, R. \& Mussa-Ivaldi, F.A., (1994). Adaptive representation of dynamics during learning of a motor task. Journal of Neuroscience, 14, 3208-3224.

Welsh, T.N. \& Elliott, D. (2000). Preparation and control of goal-directed limb movements in persons with Down syndrome. In: D.J. Weeks, R. Chua \& D. Elliott (Eds.), Perceptual-motor behavior in Down syndrome (pp. 49-70). Champaign, IL: Human Kinetics. 